\title{
Operando Investigation of the Locally Enhanced Electric Field Treatment (LEEFT) Harnessing Lightning-Rod Effect for Rapid Bacteria Inactivation
}

\section{Ting Wang}

Georgia Institute of Technology https://orcid.org/0000-0002-4658-7789

\section{Devin k. Brown}

Georgia Institute of Technology

Xing Xie ( $\nabla$ xing.xie@ce.gatech.edu )

Georgia Institute of Technology

\section{Research Article}

Keywords: Locally enhanced electric field treatment, Lightning-rod effect, Electroporation, Antimicrobial, Nanostructures, Bacteria, Operando investigation

Posted Date: August 20th, 2021

DOI: https://doi.org/10.21203/rs.3.rs-405960/v2

License: (c) (i) This work is licensed under a Creative Commons Attribution 4.0 International License. Read Full License

Version of Record: A version of this preprint was published at Nano Letters on November 4th, 2021. See the published version at https://doi.org/10.1021/acs.nanolett.1c02240. 


\section{Operando Investigation of the Locally Enhanced}

\section{Electric Field Treatment (LEEFT) Harnessing}

3 Lightning-Rod Effect for Rapid Bacteria

4 Inactivation

$5 \quad$ Ting Wang $^{1}$, Devin K. Brown ${ }^{2}$, Xing Xie ${ }^{1,2 *}$

6

$7{ }^{1}$ School of Civil and Environmental Engineering, Georgia Institute of Technology,

8 Atlanta, Georgia 30332, United States

$9{ }^{2}$ Institute for Electronics and Nanotechnology, Georgia Institute of Technology, Atlanta,

10 Georgia 30332, United States 
11 ABSTRACT. The growth of undesired bacteria causes numerous problems. Here, we show

12 that locally enhanced electric field treatment (LEEFT) can cause rapid bacteria inactivation

13 by electroporation without any side reactions. The bacteria inactivation is studied in situ at

14 the single-cell level on a lab-on-a-chip that has nanowedge-decorated electrodes. Rapid

15 bacteria inactivation occurs specifically at nanowedge tips where the electric field is

16 enhanced due to the lightning-rod effect. The mechanism study shows that the bacteria

17 inactivation is caused by electroporation induced by the locally enhanced electric field. The

18 bacteria inactivation performance depends on the strength of the enhanced electric field

19 instead of the applied voltage, and no ROS generation is detected when $>90 \%$ bacteria

20 inactivation is achieved. Quick membrane pore closure under moderate LEEFT indicates

21 that electroporation is the predominant mechanism. LEEFT only requires facile treatment

22 to achieve bacteria inactivation, which is safe for treating delicate samples and energy-

23 efficient for large scale applications. The findings in this work can provide strong supports

24 for the future applications of LEEFT.

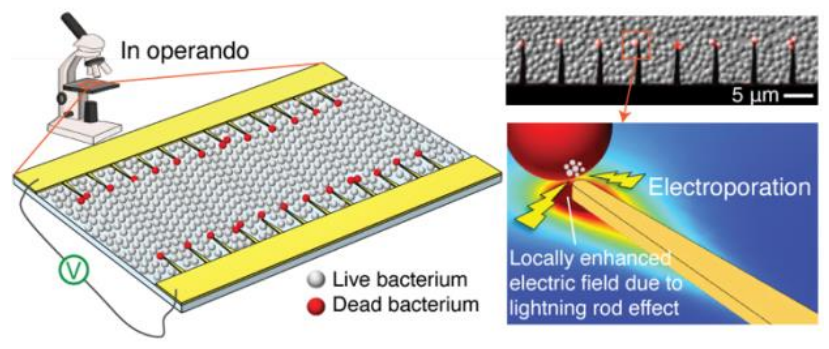

27 KEYWORDS. Locally enhanced electric field treatment, Lightning-rod effect,

28 Electroporation, Antimicrobial, Nanostructures, Bacteria, Operando investigation 
Bacteria are indispensable for both ecological systems and human bodies, but the

30 growth of undesired bacteria can also cause serious problems. Seeking approaches for

31 bacteria inactivation is an everlasting effort. Most of our current practices for bacteria

32 inactivation highly rely on the uses of chemicals, such as antibiotics for infection treatment,

33 chlorine for water disinfection, antiseptics for food preservation, and chemical anti-fouling

34 agents. They have been effectively inactivating bacteria, but caused new problems: overuse

35 of antibiotics has already raised the concern of antibiotic resistance, ${ }^{1}$ chlorination generates

36 disinfection by-products (DBPs) that can be carcinogenic, ${ }^{2}$ food antiseptics and anti-

37 fouling agents themselves may be harmful to human health or the environment.

38 Effective physical processes, such as thermo/ultraviolet radiation, ${ }^{3,} 4$ acoustic

39 vibration, ${ }^{5,6}$ microwave, ${ }^{7}$ and electric field treatment (EFT), ${ }^{8}$ can be superior alternatives

40 to chemical approaches for bacteria inactivation, although many of them suffer from high

41 capital cost or energy consumption. Among these processes, the EFT is increasingly

42 finding applications in food preservation and water disinfection. ${ }^{9-12}$ The EFT aims to

43 inactivate bacteria by electroporation: when a cell is exposed to a strong electric field, an

44 induced transmembrane voltage (TMV) will cause pore formation on the lipid bilayer

45 membrane, ${ }^{13-15}$ and when this external electric field is strong enough, the membrane

46 damage, i.e., the pores, will become lethal to the bacterial cells. ${ }^{10}$ The lethal electroporation

47 threshold was found to be between $10 \sim 35 \mathrm{kV} / \mathrm{cm} .{ }^{16}$ Typically, in order to achieve the

48 strong enough electric field, the EFT processes will require high applied voltages (e.g.,

$4923 \mathrm{kV}$ to achieve $35 \mathrm{kV} / \mathrm{cm}$ on the electrodes with $0.65 \mathrm{~cm}$ distance), ${ }^{17}$ which leads to

50 safety issues, side reactions, and high energy consumption. 
A strategy to realize the high electric field strength with lower voltages is to decorate

52 the electrodes with sharp objects, such as nanowires or nanowedges. Attributed to the

53 lightning-rod effect, the electric field near the tips could be largely enhanced depending on

54 the aspect ratio of the electrode decorations. ${ }^{18}$ As a result, even with relatively low applied

55 voltages, the locally enhanced electric field can still build up the transmembrane voltage

56 that is sufficient to cause irreversible electroporation and bacteria inactivation. Although

57 this concept has been claimed as the predominant mechanism for bench-scale EFT water

58 disinfection devices equipped with nanowire-modified electrodes, ${ }^{19-28}$ direct demonstration

59 of lightning-rod effect for bacteria inactivation, especially at the single-cell level, is not yet

60 done. Here, we conduct locally enhanced EFT (LEEFT) on a lab-on-a-chip device that has

61 nanowedge-modified electrodes and investigate the microbial inactivation process in situ.

62 Results show that the bacteria located at the tips of nanowedges on both positive and

63 negative electrodes are rapidly inactivated with the voltages that are not sufficient to kill

64 bacteria in bulk. Electroporation induced by the locally enhanced electric field attributed

65 to the lightning-rod effect is demonstrated to be the predominant mechanism for this

66 bacteria inactivation.

67 Lab-on-a-chip has been intensively used for operando investigation of microbiology

68 related processes. ${ }^{13}, 29,30$ We developed a lab-on-chip device with gold nanowedges

69 fabricated on both positive and negative electrodes (Fig. 1a \& Fig. S1). The gap between

70 the two electrodes is $50 \mu \mathrm{m}$. The length and thickness of the nanowedge are $8 \mu \mathrm{m}$ and 200

$71 \mathrm{~nm}$, respectively. The width of the nanowedge tip is $200 \mathrm{~nm}$, and it gradually increases to

$721 \mu \mathrm{m}$ to allow a steadier connection to the bulk electrode. This is the default chip design

73 for our experiments unless otherwise stated. When an $18 \mathrm{~V}$ voltage is applied to the two 
74 electrodes, the electric field near the nanowedge tips will be enhanced due to the lightning-

75 rod effect, which is simulated using COMSOL Multiphysics (Fig. 1b).

76 The chip was pre-coated with poly-L-lysine, and the model bacteria Staphylococcus 77 epidermidis (S. epidermidis) cells were uniformly attached on the chip (Fig. 1c). Since the 78 bacteria cells are negatively charged, they are firmly immobilized by the positively charged 79 poly-L-lysine and will remain at the original place even after electrical pulses. Live-and80 dead cell distinguishing stain propidium iodide (PI) was added in the deionized water (DI 81 water) medium before treatment (See experimental setup in Fig. S2a). After 500,000 82 electrical pulses at $18 \mathrm{~V}$ with $2 \mu$ s pulse width and $100 \mu$ s period are applied (denoted as $8318 \mathrm{~V} / 2 \mu \mathrm{s} / 100 \mu \mathrm{s} / 500,000$ pulses, see the waveform in Fig. S3), the bacteria at the tips of 84 nanowedges on both positive and negative electrodes show red fluorescence of the PI stain, 85 indicating cell membrane damage, while cells anywhere else are intact (Fig. 1d). The 86 zoom-in image clearly shows that only the cells located very close to the nanowedge tips

87 are damaged, which is consistent with the electric field enhancement pattern (Fig. 1b). By

88 comparison, for the electrodes that have no nanowedge modification but a smaller gap of

$8934 \mu \mathrm{m}$, hardly any cells are affected (Fig. S4), suggesting that this electrical treatment is 90 not sufficient to kill bacteria in bulk or on electrode edge. Therefore, LEEFT can cause 91 bacteria inactivation with lower applied voltages than in bulk EFT.

92 The bacteria damage process was observed in real-time. The onset position of PI 93 fluorescence indicates that the cell membrane damage takes place at the position adjacent 94 to the nanowedge tip, where the nano-enhanced electric field has the highest strength. The 95 circled bacteria cells at the nanowedge tips on the negative electrode (Fig. 1e) and positive 96 electrode (Fig. 1f) do not show fluorescence before the treatment ( $0 \mathrm{~s})$. The arrows indicate 
97 the location where the cell membrane is adjacent to the nanowedge tip. After the treatment

98 starts, the red fluorescence of PI stain first originates from the adjacent point indicated by

99 the arrows (shown in $0.1 \mathrm{~s}, 0.2 \mathrm{~s}$, and $0.4 \mathrm{~s}$ ), suggesting that the part of the cell membrane

100 subjected to the strongest electric field will be perforated first.

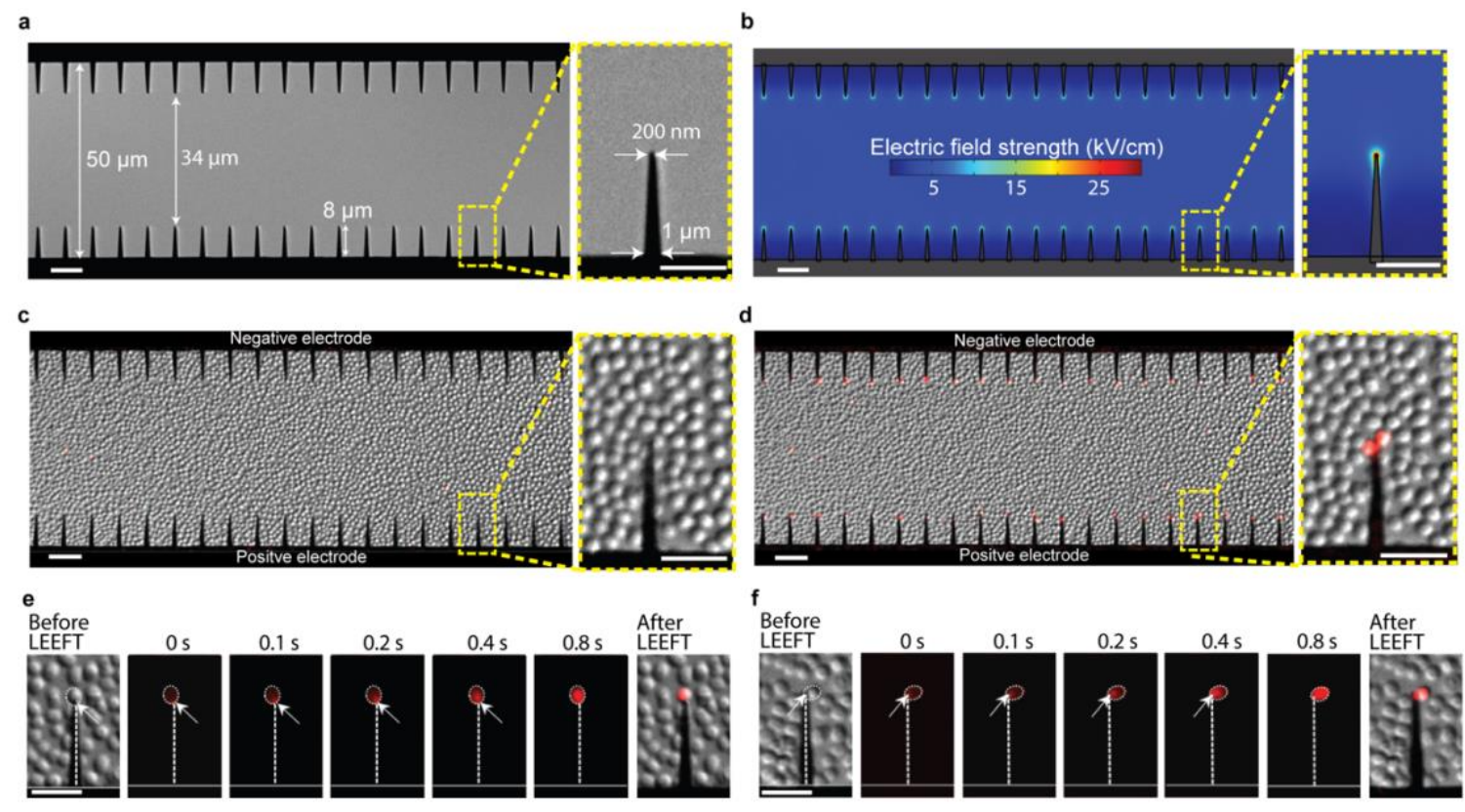

102 Figure 1. Bacteria damage in LEEFT. (a) Microscopy image of the lab-on-a-chip device. (b) The

103 nano-enhanced electric field at the nanowedge tips at $18 \mathrm{~V}$ applied voltage. (c \& d) Microscopy

104 images of the immobilized bacteria cells before (c) and after (d) LEEFT treatment. Scale bars are

$10510 \mu \mathrm{m}$ in normal images and $5 \mu \mathrm{m}$ in the zoom-in images. (e \& f) PI fluorescence onset indicating

106 pore formation position of a cell at nanowedge tip on negative electrode (e) and positive electrode

107 (f). The arrows indicate the position of the cell membrane adjacent to the nanowedge tip, which is

108 also the onset position of PI fluorescence. Scale bars are $5 \mu \mathrm{m}$.

110 The bacteria damage occurs rapidly in LEEFT, which can be seen from the video

111 Movie S1. To figure out how fast it is, different effective treatment time (i.e., the total time 
112 that the applied voltage is not zero, equals to pulse width $\times$ pulse number) was tested by

113 applying different pulse numbers of $2 \mu \mathrm{s} / 100 \mu$ s pulses. Under $30 \mathrm{~V}$ and $18 \mathrm{~V}$ applied

114 voltage, $0.1 \mathrm{~ms}$ and $1 \mathrm{~ms}$ of effective treatment times are long enough to achieve $>80 \%$

115 bacteria damage (represented by the percentage of nanowedges inducing bacteria damage

116 at tips), respectively, indicating that bacteria damage in LEEFT is very rapid (Fig. 2a).

117 Under relatively lower applied voltages (14 V and $10 \mathrm{~V}$ ), bacteria damage efficiency stays

118 at low percentages up to $1 \mathrm{~s}$ of effective treatment time, suggesting that the limiting factor

119 of the lower bacteria damage is the applied voltage rather than treatment time (Fig. 2a).

120 Therefore, the bacteria damage efficiency at different applied voltages with $1 \mathrm{~s}$ effective

121 treatment time were tested. In case the cell damage was reversible, we conducted parallel

122 experiments but stained the cells with PI 2 hours after treatment. Since reversible

123 membrane damages should already recover after 2 hours, the cells stained with PI are

124 considered inactivated. ${ }^{14}$ There is no significant difference between the efficiency of cell

125 damage and inactivation, except that cell inactivation is even higher especially at low

126 voltages (Fig. 2b), which is due to random cell inactivation in all cells after 2 hours. The

127 efficiency shows a positive correlation with the applied voltage (Fig. 2b). It starts at a low

128 voltage of $10 \mathrm{~V}$, and $20 \mathrm{~V}$ is already high enough to achieve bacteria inactivation for almost

129 all nanowedges, and there is no significant difference between the positive and negative

130 electrodes. This result indicates that with $1 \mathrm{~s}$ effective treatment time, most cell damage is

131 irreversible, leading to cell inactivation. The phenomenon that damaged bacteria lost cell

132 integrity and decayed after stored in nutrient broth at $35^{\circ} \mathrm{C}$ for 6 hours further confirms

133 cell inactivation (Fig. S5). Therefore, when $1 \mathrm{~s}$ effective time is used, we consider the cell

134 inactivation efficiency is approximately the same with cell damage. 
135 The high aspect ratio of the nanowedges is important to LEEFT, indicated by the 136 control experiments with different chip designs (Figs. 2c - f). After treatment of 18 V/2 $137 \mu \mathrm{s} / 100 \mu \mathrm{s} / 500,000$ pulses, the nanowedges with $200 \mathrm{~nm}$ width at the tip (Figs. 2c \& e) and $1388 \mu \mathrm{m}$ length (Figs. 2d \& f) show a much higher efficiency of bacteria inactivation than 139 other wider or shorter electrode modifications. LEEFT also works for free-moving bacteria 140 cells suspended in the medium. Syto 9 and PI-stained S. epidermidis are suspended in DI 141 water before the treatment (Fig. 2g upper. See the experimental setup in Fig. S2b). During 142 the LEEFT (18 V/2 $\mu \mathrm{s} / 100 \mu \mathrm{s} / 500,000$ pulses), bacteria cells are attracted toward the 143 nanowedges on both positive and negative electrodes, especially to the tips. Subsequently, 144 those near the tips get inactivation, indicated by switching from green fluorescence of Syto 1459 to red fluorescence of PI (Movie S2, Fig. 2g lower). As the bacteria cells are negatively 146 charged in DI water, most of them accumulated at the positive electrode because of the 147 electrophoretic force. Some cells are attracted to the nanowedge tips on the negative 148 electrode, which is probably due to the strong dielectrophoretic force induced by the 149 electric field enhancement near the tips. ${ }^{27,31}$ Two other kinds of bacteria, Bacillus subtilis 150 (B. subtilis, Gram +) and Escherichia coli (E. coli, Gram -), are also tested, which show 151 similar transport and inactivation phenomena with S. epidermidis (Figs. 2h \& i, Movies 152 S3 \& S4), suggesting that LEEFT could be a wide spectrum bacteria inactivation method. 


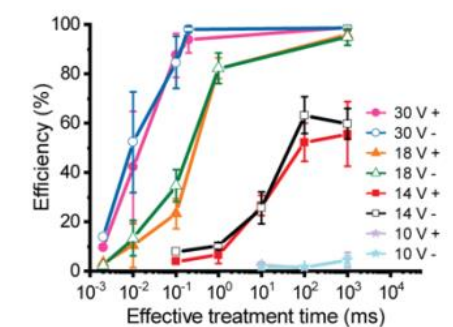

c

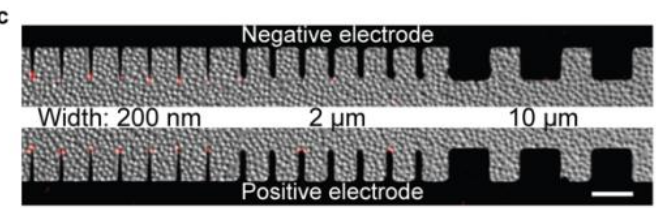

e

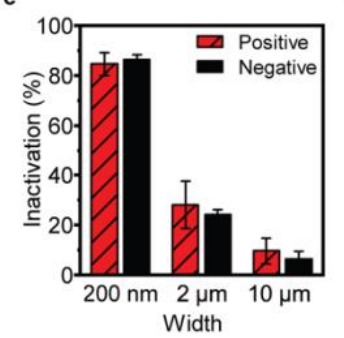

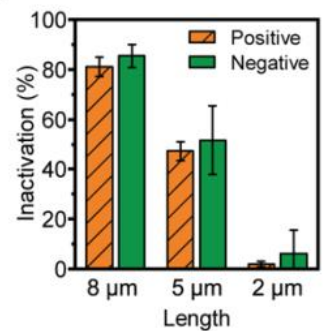

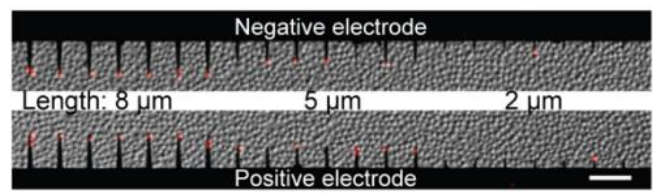

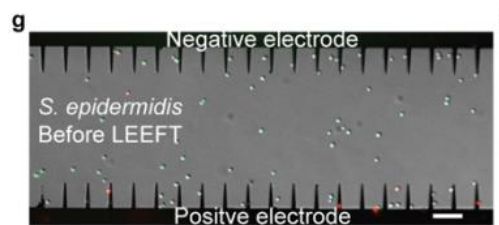
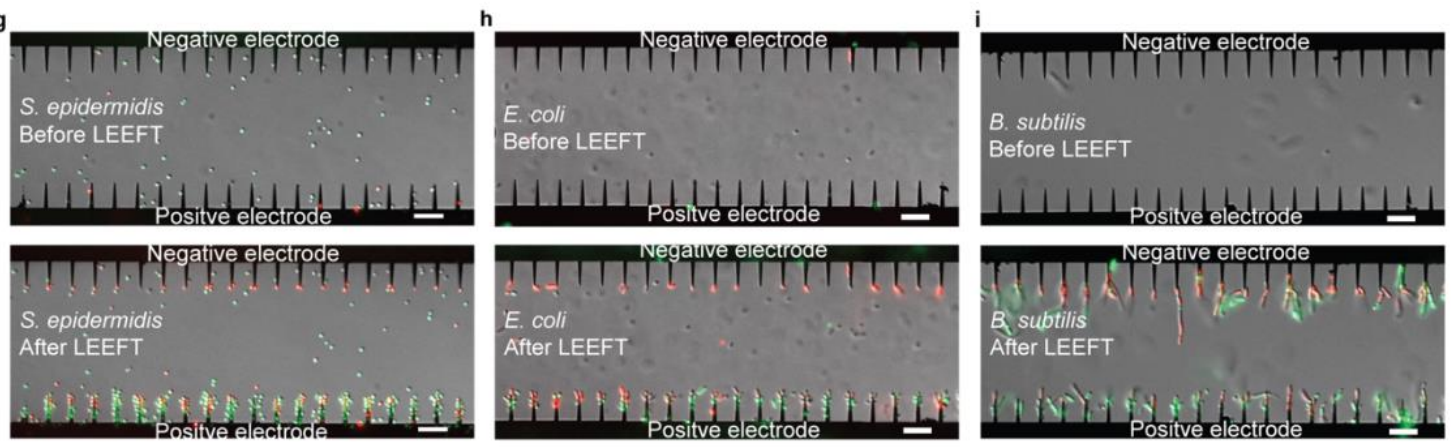

154 Figure 2. Bacteria inactivation characterization in LEEFT. (a) Bacteria damage efficiency (i.e., the

155 percentage of nanowedges inducing bacteria damage) with different effective treatment time.

156 Positive and negative electrodes are denoted as + and -, respectively. (b) Bacteria damage and

157 inactivation efficiency versus the applied voltage with $1 \mathrm{~s}$ effective treatment time. $(\mathrm{c}-\mathrm{f})$ Bacteria

158 inactivation with wedges of different width (c \& e) and different length (d \& f). (g - i) Microscopy

159 images of different kinds of bacteria in suspension before (upper) and after (lower) the LEEFT. (g)

160 S. epidermidis. (h) E. coli. (i) B. subtilis. The scale bars are $10 \mu \mathrm{m}$.

In LEEFT, only the bacteria located near nanowedge tips are inactivated, while

163 bacteria in bulk are not affected. This pattern is consistent with the electric field

164 enhancement of nanowedges due to the lightning-rod effect, suggesting that irreversible 
165 electroporation induced by the enhanced electric field is the most possible mechanism for

166 bacteria inactivation. Here, we investigated the mechanism, and the evidence collected is

167 discussed below.

168 First, we found that the bacteria inactivation depends on the strength of the nano-

169 enhanced electric field rather than the applied voltage. Chips of different positive/negative

170 electrode gaps $(25 \mu \mathrm{m}, 50 \mu \mathrm{m}, 100 \mu \mathrm{m})$ and with nanowedges of different intervals $(0.8$

$171 \mu \mathrm{m}, 4 \mu \mathrm{m}, 40 \mu \mathrm{m}$ ) were tested for bacteria inactivation. The strength of the nano-enhanced

172 electric field is reversely proportional to the gap between the two electrodes (Figs. S6a, b

$173 \boldsymbol{\&} \mathbf{c})$. Therefore, with the same applied voltage, chips with a smaller gap achieve a higher

174 percentage of bacteria inactivation (Fig. 3a \& Fig. S6d). Similarly, because of the stronger

175 lightning-rod effect for electric-field enhancement (Figs. S7a $\&$ b), the nanowedges with

176 a larger interval in between could achieve higher bacteria inactivation under the same

177 applied voltage (Fig. 3b \& Fig. S7c). When all the results are analyzed, the percentage of

178 bacteria inactivation at the tips of nanowedges shows a positive correlation with the electric

179 field strength (Fig. 3c left), but not with the applied voltages (Fig. 3c right). This result

180 indicates that the bacteria inactivation is attributed to the nano-enhanced electric field.

181 The bacteria inactivation is not attributed to reactive oxygen species (ROS). Although

182 inducing ROS is a commonly used antimicrobial method, it has some side effects, including

183 generating by-products or causing sample damage when high sample quality needs to be

184 preserved, such as blood sample or liquid food. Electric-field treatment systems could

185 generate ROS, especially under high voltages or long treatment times. To test if the bacteria

186 inactivation is attributed to ROS damage, DCFH-DA stain was used to detect ROS

187 generation. ${ }^{32,33}$ In the experiment group with $30 \mathrm{~V} / 2 \mu \mathrm{s} / 100 \mu \mathrm{s} / 100,000$ pulses treatment, 
188 DCFH-DA stained cells show no fluorescence (Fig. 3d upper), suggesting no ROS 189 generation. Meanwhile, $>90 \%$ bacteria inactivation is achieved (Fig. 3d lower \& 190 experiment group, no DMSO in Fig. 3h), indicating that this bacteria inactivation is not 191 due to ROS damage. To confirm this ROS detection method is valid, we intentionally 192 induced ROS generation with a much longer pulse width in the positive control (20 V/200 $193 \mu \mathrm{s} / 10 \mathrm{~ms} / 1000$ pulses). The significant green fluorescence of DCFH-DA stained cells

194 shows that ROS is generated near the positive electrode (Fig. 3e upper). The positive 195 electrode shows more inactivated bacteria at each nanowedge tip than the experiment group 196 and negative electrodes (Fig. 3e lower \& positive control, no DMSO in Fig. 3h), which 197 could be attributed to the ROS damage.

198 To further confirm that the bacteria inactivation at $30 \mathrm{~V} / 2 \mu \mathrm{s} / 100 \mu \mathrm{s}$ is not due to ROS, 199 a ROS scavenger, DMSO, was added to the medium at 15\% (w/w) to quench ROS and 200 protect bacteria from its damage. ${ }^{34}$ In the positive control group, the bacteria at the positive 201 electrodes are largely protected by DMSO (Fig. 3g \& positive control in Fig. 3h), proving 202 that $15 \%$ DMSO is able to protect bacteria from ROS damage. In the experiment group, 203 even with the ROS scavenger, the bacteria inactivation percentage and inactivated cell 204 number are not affected (Fig. 3f \& experiment group in Fig. 3h), which further confirms 205 that the bacteria inactivation is not due to ROS damage. 
a

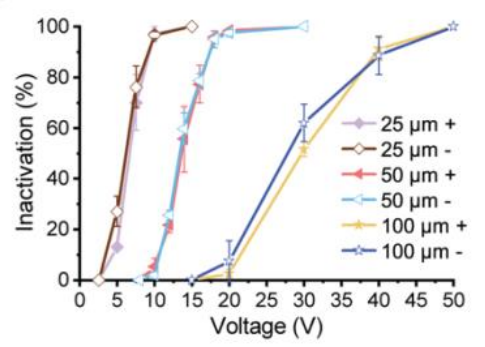

b

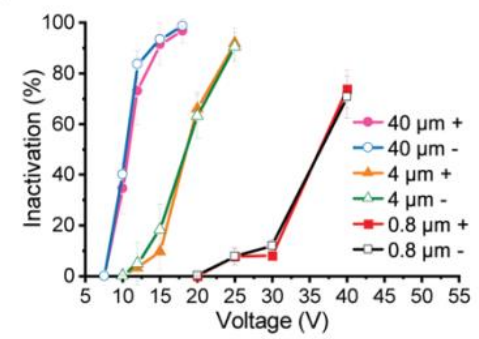

c

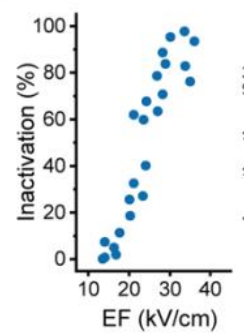

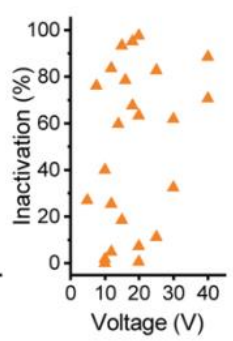

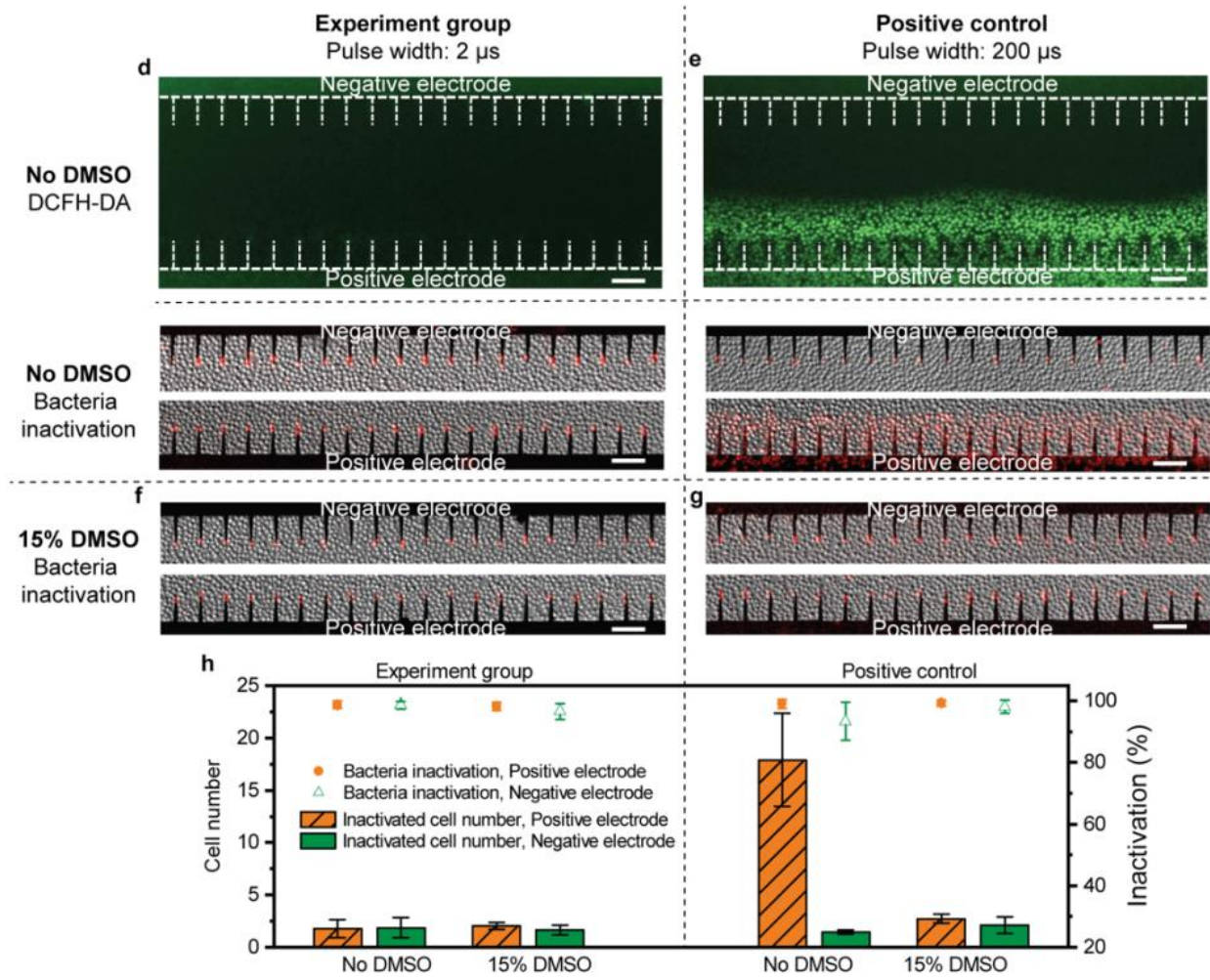

207 Figure 3. Antibacterial mechanism investigations. (a) Bacteria inactivation on chips of

208 different gaps between positive and negative electrodes. (b) Bacteria inactivation on chips

209 of different intervals between nanowedges. (c) Relationship between the bacteria

210 inactivation and the electric field strength (EF) at the tip of the nanowedge ( $0.1 \mu \mathrm{m}$ away

211 from the nanowedge tip) (left), and the applied voltage (right). (d) Fluorescence of DCFH-

212 DA-stained cells (upper), and bacteria inactivation with no DMSO lower in experiment

213 group (lower). (e) Fluorescence of DCFH-DA-stained cells (upper), and bacteria

214 inactivation in positive control group (lower). Green fluorescence indicates ROS 
215 generation. (f \& g) Bacteria inactivation in (f) experiment group and (g) positive control

216 with 15\% DMSO. (h) Bacteria inactivation percentage and average inactivated cell number

217 at each nanowedge tip. Scale bars are $10 \mu \mathrm{m}$.

218 The third piece of evidence is quick cell membrane recovery after LEEFT, which also

219 supports that electroporation is the main bacteria inactivation mechanism. Reversible

220 electroporation is a phenomenon that pores formed on the lipid bilayer membrane reseal

221 automatically after the electric field is removed. It occurs when the cell is exposed to a

222 relatively weaker electric field or shorter treatment time than irreversible electroporation. ${ }^{14}$

223 The PI fluorescence intensity of four cells under $14 \mathrm{~V} / 2 \mu \mathrm{s} / 100 \mu$ s intermittent treatment

224 shows that when the treatment is on (red shadow, 10,000 pulses for $1 \mathrm{~s}$ total time), the

225 fluorescence increases, which means pore formation and PI dye inflow (Fig. 4a). When the

226 treatment is removed (gray shadow, $5 \mathrm{~s}$ ), the fluorescence stops rising immediately,

227 suggesting that the pores close and the membrane regains its integrity after the treatment

228 stops (Fig. 4a). This kind of quick cell membrane recovery is a common phenomenon in

229 reversible electroporation, ${ }^{13,14}$ but is hard to find in other kinds of membrane damages,

230 such as direct oxidation. Therefore, quick pore reseal is strong evidence for reversible

231 electroporation.

232 Reversible electroporation was also tested using a double staining method with

233 SYTOX Green and PI, which can only enter cells with compromised membrane. ${ }^{35}$ SYTOX

234 Green is first added to the medium (Time point 1, Fig. 4b). After the LEEFT is applied,

235 perforated cells are stained with SYTOX Green and show green fluorescence (Time point

236 2, Fig. 4b). After 10 minutes, PI is added, which could only stain the cells that still have

237 compromised membrane. Thus, the cells that are not stained with PI are considered as 
238 having reversible pores (Time point 3, Fig. $\mathbf{4 b}$ ). With a relatively low applied voltage at 14

$239 \mathrm{~V}(2 \mu \mathrm{s} / 100 \mu \mathrm{s} / 20,000$ pulses $)$, some already perforated cells could not then be stained with

$240 \mathrm{PI}$, indicating the pores formed on the cell membrane are reversible (Fig. 4c). While under

241 a high applied voltage at $80 \mathrm{~V}(1 \mu \mathrm{s} / 1 \mathrm{~ms} / 10$ pulses $)$, almost all cell perforation is

242 irreversible (Fig. S8). This phenomenon conforms to the feature of electroporation,

243 indicating that electroporation is the predominant mechanism for bacteria inactivation in

244 LEEFT. Note that when $1 \mathrm{~s}$ effective treatment time (500,000 pulses) is used, there is no

245 significant reversible damage as shown in Fig. 2b. It is mainly due to the longer treatment

246 time, which greatly increases the cell death possibility. It is also possible that although

247 reversible pores close after LEEFT, they damage cell function, which leads to cell 248 inactivation ultimately.

a

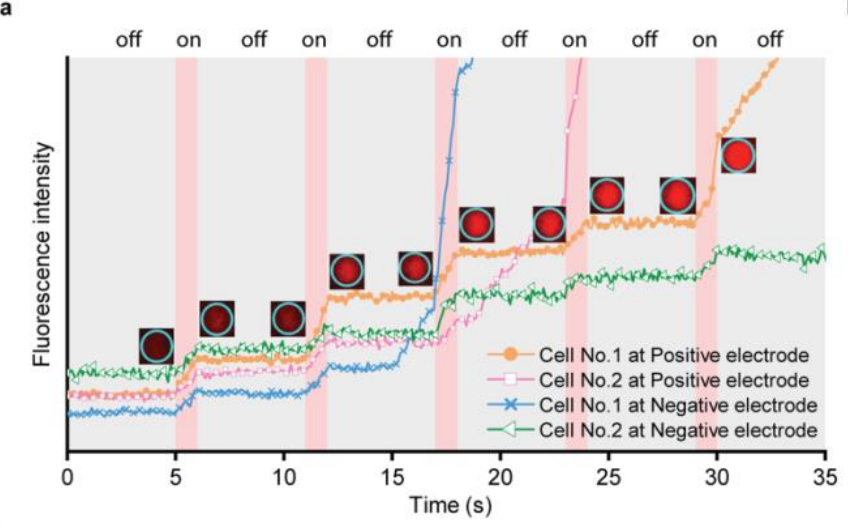

Negative electrode
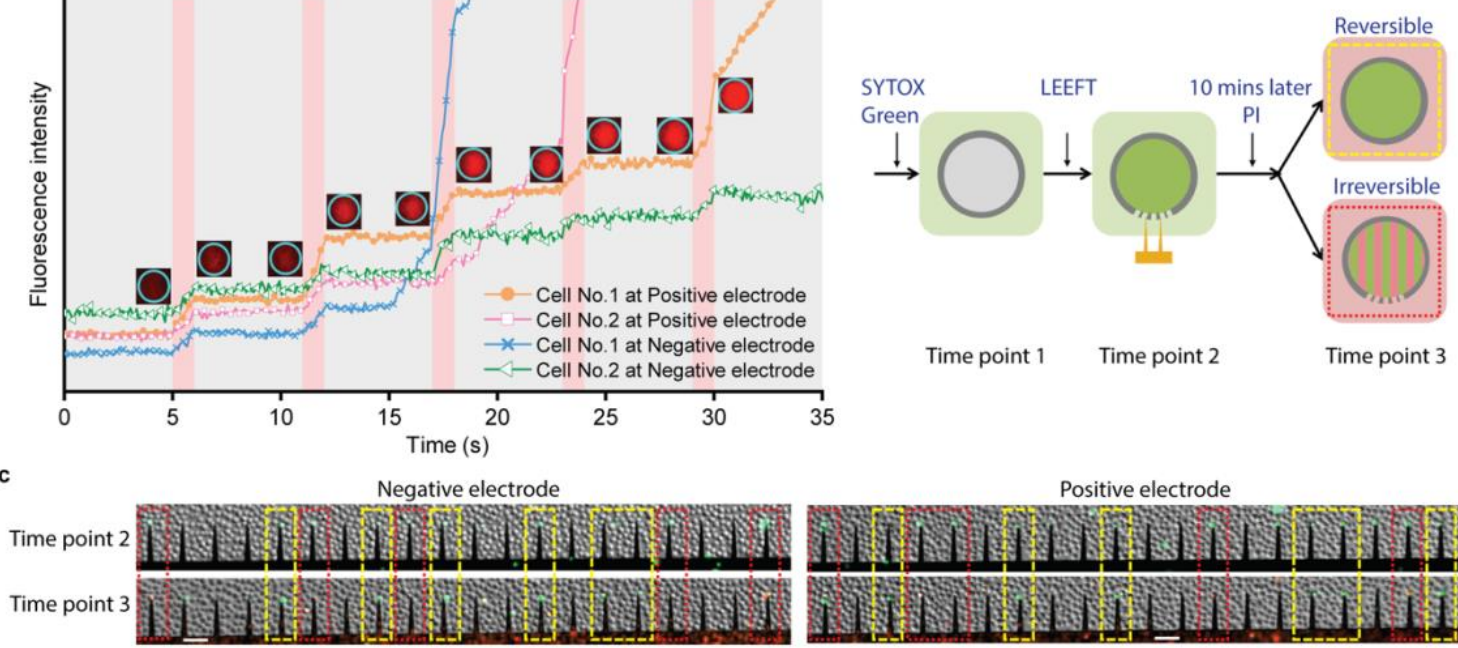

Positive electrode

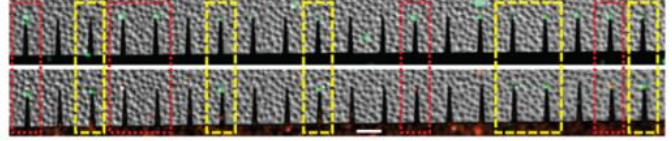

250 Figure 4. Detection of reversible electroporation. (a) Increase of PI stain fluorescence of four cells

251 at nanowedge tips on positive and negative electrodes, respectively, with intermittent LEEFT. The

252 red shadow indicates that the LEEFT is on, which are $14 \mathrm{~V} / 2 \mu \mathrm{s} / 100 \mu \mathrm{s} / 10,000$ pulses for $1 \mathrm{~s}$ total

253 time. The gray shadow indicates that the LEEFT is off, which is $0 \mathrm{~V}$ for $5 \mathrm{~s}$. The inserted images

254 show Cell No. 1 at the positive electrode (orange spheres). (b) Schematic of double staining method 
255 with SYTOX Green and PI for reversible electroporation detection. (c) Microscopy images

256 showing reversible electroporation under $14 \mathrm{~V}$. The cells inside the yellow frames had reversible

257 pores on membrane since they are stained with SYTOX Green at Time point 2 but are not stained

258 with PI at Time point 3. The cells inside the red frames have irreversible pores since they are first

259 stained with SYTOX Green and then stained with PI. Scale bars are $5 \mu \mathrm{m}$.

261 Due to the lightning-rod effect, the electric field at tips of metal rods with a high aspect

262 ratio will be greatly enhanced compared to that in bulk. Therefore, this strong electric field

263 could be sufficient to charge cell membrane, cause irreversible electroporation, and kill

264 bacteria even under lower applied voltages. Although bench-scale LEEFT for water

265 disinfection was developed based on this concept, the mechanism was only supported by

266 control experiments done with electrodes with/without nanowire modifications. ${ }^{19,20}$ There

267 was no direct evidence confirming that the bacteria were inactivated due to the locally

268 enhanced electric field and electroporation. The results achieved in this study provide

269 important evidence on the mechanism. Firstly, only the bacteria in the area of the locally

270 enhanced electric field are inactivated while others in bulk are intact (Fig. 1b \& d). The

271 inactivation percentage shows a positive correlation with the strength of the locally

272 enhanced electric field instead of the applied voltage (Fig. 3c). Furthermore, when $>90 \%$

273 bacteria inactivation is achieved with LEEFT at $30 \mathrm{~V} / 2 \mu \mathrm{s} / 100 \mu \mathrm{s}$, there is no significant

274 ROS generation (Fig. 3d), indicating this bacteria inactivation is not due to ROS damage.

275 Reversible electroporation is detected under relatively low applied voltage (Fig. 4),

276 suggesting that LEEFT could induce electroporation, and irreversible electroporation

277 causing bacteria inactivation could be dominant at higher voltages. 
It is worth noticing that electric field enhancement by nanowedges is the same for both

279 positive and negative electrodes (Fig. S6 \& S7). Consistently, all the bacteria inactivation

280 phenomena discussed above do not show a significant difference between positive and

281 negative electrodes. An electrochemical disinfection study reported that anode induced

282 significantly higher bacteria inactivation than cathode, suggesting that electrical reduction

283 should not cause the same level of cell damage as electrical oxidation. ${ }^{36}$ Our positive

284 control group for ROS detection also confirms that (Fig. 3e). Therefore, the same

285 phenomenon on both electrodes found in this work indicates that electrical

286 oxidation/reduction should not be the mechanism causing bacteria inactivation. Besides

287 that, metal ions should not play a role in bacteria inactivation, neither. Since gold is

288 electrochemically stable, under the very short $2 \mu$ s pulses at voltages lower than $30 \mathrm{~V}$, there

289 should be no significant gold ion release. Therefore, electroporation is demonstrated as the

290 predominant mechanism causing bacteria inactivation in LEEFT.

291 Since electroporation is the predominant mechanism for bacteria inactivation, the

292 induced transmembrane voltage (TMV), which is the increased potential difference across

293 the cell membrane resulting from exposure to an external electric field, was analyzed

294 theoretically using finite element simulation to compare LEEFT and bulk EFT. Both the

295 on-chip system like the one used in this work (Fig. 5) and a 3D system with standing

296 nanowire (Fig. S9) were simulated. Two cells in LEEFT and bulk EFT respectively are

297 compared, which is Cell No. 1 located at the nanowedge tip (Fig. 5a left), and Cell No. 2

298 located between two electrodes without nanowedge (Fig. 5a right). The simulation results

299 show that the voltage drop across the membrane, i.e., the electric field, is greatly enhanced

300 at Cell No. 1 near the nanowedge tip (Fig. 5b left) compared to Cell No. 2 (Fig. 5b right). 
301 The maximum TMVs of the two cells show that with the same applied voltage, the cell No.

3021 in LEEFT located at the nanowire tip can achieve around 7.5 times higher TMV than cell

303 No. 2 in bulk EFT (Fig. 5c), indicating that much lower voltage could be applied to achieve

304 the same level of TMV on cells in LEEFT than bulk EFT.

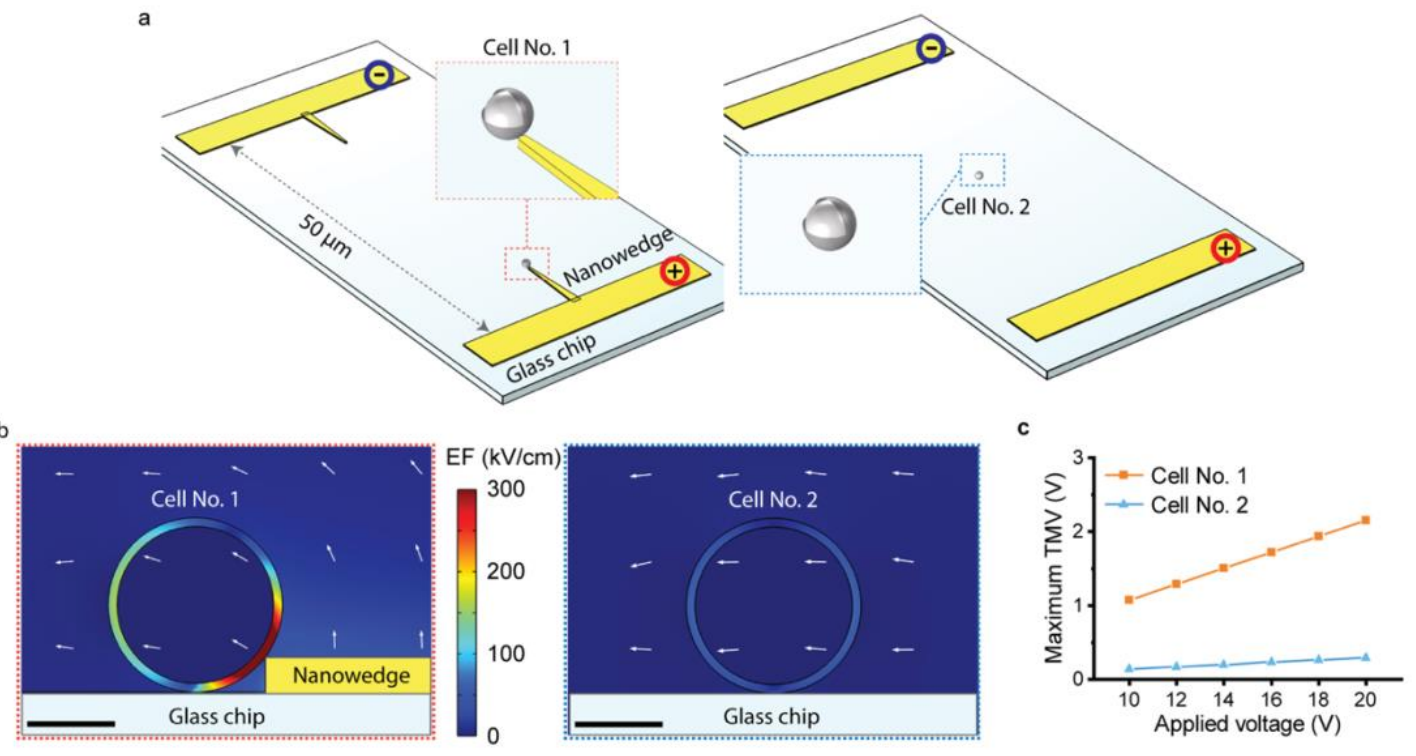

b

306 Figure 5. Theoretical analysis of cell TMV in LEEFT and bulk EFT. (a) Simulation set up

307 for LEEFT (left) and bulk EFT (right). (b) Left view of the middle cutting plane showing

308 the electric field across the cell membrane of cell No. 1 in LEEFT (left) and cell No. 2 in

309 bulk EFT under $20 \mathrm{~V}$ applied voltage (right). The arrows indicate the direction of the

310 electric field. The scale bars are $0.5 \mu \mathrm{m}$. (c) Maximum TMV on cell No. 1 and cell No. 2

311 under different applied voltages.

313 This work is a single-cell level proof-of-concept study of LEEFT, which directly

314 proves that combining lighting rod effect and electroporation can be used for antimicrobial

315 purpose. We are optimistic that LEEFT technique can be developed to have plenty of 
316 specific applications. LEEFT effectively kills bacteria with mild treatment conditions

317 without causing side effects, making it suitable for high-quality sample processing, such as

318 liquid food or blood sample, and also safe for medical applications, such as for wound

319 healing. Lower voltages and short pulses make it is an energy-efficient approach that is

320 applicable for large-scale treatment processes, such as water treatment. Since the bacteria

321 inactivation is highly localized, it is perfect for surface treatment, such as biofilm and

322 biofouling prevention, and it can also find applications in continuous flow system, since

323 bacteria could be attracted to the nanowedge tips and get inactivated. The as-shown rapid

324 cell damage and the effectiveness of both electrodes further improve its efficiency. Since

325 electroporation targets the phospholipid membrane, LEEFT should work on a broad range

326 of cell types with more applications, including intracellular molecule delivery and cell 327 lysing.

328 In real applications, the efficiency of LEEFT could be further improved through 329 several ways. Electrodes all covered with nanowires could be developed to increase the 330 effective zones. The treatment system and operation process could also be improved 331 depending on different applications. For instance, fluid mixing could be introduced in

332 continuous flow systems to improve the possibility of transporting bacteria to the nanowire 333 tips. The parameters of electrical pulses, such as pulse width, directions, and voltages,

334 could be altered to effectively manipulate cells in the flow and to attract bacteria cells to 335 the nanowire tips. For large scale applications, the cost could be reduced by using cheaper 336 electrode materials, such as $\mathrm{Cu}$ electrodes with $\mathrm{CuO}$ or $\mathrm{Cu}_{3} \mathrm{P}$ nanowires synthesized on the 337 surface. The synergistic effect of electroporation with metal ions, ${ }^{37,} 38$ ozone, ${ }^{25}$ and other 338 antimicrobial reagents could also be applied to further improve the efficiency of LEEFT. 
340 Supporting information

341 Movie S1: Bacteria inactivation process in LEEFT (MP4)

342 Movie S2: Transport and inactivation of free-moving S. epidermidis cells in LEEFT

343 (MP4)

344 Movie S3: Transport and inactivation of free-moving E. coli cells in LEEFT (MP4)

345 Movie S4: Transport and inactivation of free-moving B. subtilis cells in LEEFT (MP4)

346 Materials and methods; figures S1-S9 showing the digital photo of the lab-on-a-chip, the

347 experimental setup, the waveform, the electrodes have no nanowedges as a control, the cell

348 decay after LEEFT, the chips of different electrode gap, the chips with nanowedges of

349 different interval, the irreversible electroporation at $80 \mathrm{~V}$, the simulation of TMV; screen

350 shots of Movie S1-S4 and the captions (PDF).

351

352 AUTHOR INFORMATION

353 Corresponding Author

$354 *$ Xing Xie - School of Civil and Environmental Engineering, Georgia Institute of

355 Technology, Atlanta, Georgia 30332, United States

356311 Ferst Drive NW, Room 3236, Ford ES\&T Building, Atlanta, GA 30332-0100,

357 United States

358 Email: xing.xie@gatech.edu

\section{Authors}

360 Ting Wang - School of Civil and Environmental Engineering, Georgia Institute of

361 Technology, Atlanta, Georgia 30332, United States; orcid.org/0000-0002-4658-7789 
362 Devin K. Brown - Institute for Electronics and Nanotechnology, Georgia Institute of

363 Technology, Atlanta, GA 30332, United States.

364 Author Contributions

365 X.X. and T.W. designed research. T.W. performed research. D.K.B. contributed new

366 reagents/analytic tools. T.W. and X.X. analyzed data and wrote the paper.

367 Competing Interest Statement

368 The authors declare no competing interests.

\section{Funding Sources}

370 National Science Foundation [grant numbers CBET 1845354].

372 ACKNOWLEDGMENTS

373 The authors acknowledge the financial support from the National Science Foundation

374 [grant numbers CBET 1845354]. This work was performed in part at the Georgia Tech

375 Institute for Electronics and Nanotechnology, a member of the National Nanotechnology

376 Coordinated Infrastructure (NNCI), which is supported by the National Science

377 Foundation [grant numbers ECCS-1542174]. T.W. is grateful for the financial support 378 provided by the China Scholarship Council. 
1. Neu, H. C. Science 1992, 257, (5073), 1064-1073.

2. Sedlak, D. L.; von Gunten, U. Science 2011, 331, (6013), 42-43.

3. McKinney, C. W.; Pruden, A. Environmental science \& technology 2012, 46, (24), 1339313400.

4. Hijnen, W. A. M.; Beerendonk, E. F.; Medema, G. J. Water Research 2006, 40, (1), 3-22.

5. Gao, S.; Hemar, Y.; Ashokkumar, M.; Paturel, S.; Lewis, G. D. Water research 2014, 60, $93-$ 104.

388 6. Lu, H.; Mutafopulos, K.; Heyman, J. A.; Spink, P.; Shen, L.; Wang, C.; Franke, T.; Weitz, D. A. 389 Lab on a Chip 2019, 19, (24), 4064-4070.

390 7. Plazas-Tuttle, J.; Das, D.; Sabaraya, I. V.; Saleh, N. B. Environmental Science: Nano 2018, 5, 391 (1), 72-82.

392 8. Grahl, T.; Markl, H. Applied Microbiology and Biotechnology 1996, 45, (1-2), 148-157.

393 9. Barba, F. J.; Parniakov, O.; Pereira, S. A.; Wiktor, A.; Grimi, N.; Boussetta, N.; Saraiva, J. A.;

394 Raso, J.; Martin-Belloso, O.; Witrowa-Rajchert, D. Food Research International 2015, 77, 773-

395798.

396 10. Kotnik, T.; Frey, W.; Sack, M.; Meglič, S. H.; Peterka, M.; Miklavčič, D. Trends in

397 biotechnology 2015, 33, (8), 480-488.

398 11. Bendicho, S. I.; Barbosa-Cánovas, G. V.; Martín, O. Trends in Food Science \& Technology

399 2002, 13, (6-7), 195-204.

400 12. Gusbeth, C.; Frey, W.; Volkmann, H.; Schwartz, T.; Bluhm, H. Chemosphere 2009, 75, (2),

$401 \quad 228-233$.

402 13. Sengel, J. T.; Wallace, M. I. Proceedings of the National Academy of Sciences 2016, 113,

403 (19), 5281-5286.

404 14. Kotnik, T.; Rems, L.; Tarek, M.; Miklavčič, D. Annual review of biophysics 2019, 48, 63-91.

405 15. Tieleman, D. P.; Leontiadou, H.; Mark, A. E.; Marrink, S.-J. Journal of the American Chemical

406 Society 2003, 125, (21), 6382-6383.

407 16. Wang, T.; Chen, H.; Yu, C.; Xie, X. Environment international 2019, 132, 105040.

408 17. Yildiz, S.; Pokhrel, P. R.; Unluturk, S.; Barbosa-Canovas, G. V. Food Research International

409 2021, 140.

410 18. Rojas-Chapana, J. A.; Correa-Duarte, M. A.; Ren, Z.; Kempa, K.; Giersig, M. Nano Letters $4112004,4,(5), 985-988$.

412 19. Liu, C.; Xie, X.; Zhao, W.; Liu, N.; Maraccini, P. A.; Sassoubre, L. M.; Boehm, A. B.; Cui, Y. $413 \quad$ Nano letters 2013, 13, (9), 4288-4293.

414 20. Huo, Z.-Y.; Xie, X.; Yu, T.; Lu, Y.; Feng, C.; Hu, H.-Y. Environmental science \& technology 415 2016, 50, (14), 7641-7649.

416 21. Huo, Z.-Y.; Zhou, J.-F.; Wu, Y.; Wu, Y.-H.; Liu, H.; Liu, N.; Hu, H.-Y.; Xie, X. Journal of

417 Materials Chemistry A 2018, 6, (39), 18813-18820.

418 22. Huo, Z.-Y.; Liu, H.; Wang, W.-L.; Wang, Y.-H.; Wu, Y.-H.; Xie, X.; Hu, H.-Y. Journal of materials 419 chemistry $A$ 2019, 7, (13), 7347-7354.

420 23. Huo, Z.-Y.; Kim, Y.-J.; Suh, I.-Y.; Lee, D.-M.; Lee, J. H.; Du, Y.; Wang, S.; Yoon, H.-J.; Kim, S.-W. $421 \quad$ Nature Communications 2021, 12, (1), 1-11.

422 24. Huo, Z.-Y.; Liu, H.; Yu, C.; Wu, Y.-H.; Hu, H.-Y.; Xie, X. Chemical Engineering Journal 2019, 423 369, 1005-1013.

424 25. Zhou, J.; Wang, T.; Xie, X. Environmental Science \& Technology 2020, 54, (21), 14017-14025.

425 26. Zhou, J.; Wang, T.; Chen, W.; Lin, B.; Xie, X. Environmental Science: Nano 2020, 7, (2), 397-

426403. 
27. Zhou, J.; Wang, T.; Yu, C.; Xie, X. Frontiers of Environmental Science \& Engineering 2020, 14, 428 1-12.

429 28. Zhou, J.; Yu, C.; Wang, T.; Xie, X. Journal of Materials Chemistry A 2020, 8, (25), $12262-$

43012277.

431 29. Jiang, X.; Hu, J.; Petersen, E. R.; Fitzgerald, L. A.; Jackan, C. S.; Lieber, A. M.; Ringeisen, B. R.; Lieber, C. M.; Biffinger, J. C. Nature communications 2013, 4, (1), 1-6.

30. Wang, T.; Yu, C.; Xie, X., Microfluidics for Environmental Applications. Springer Berlin Heidelberg: Berlin, Heidelberg, 2020; pp 1-24.

2004, 76, (6), 1571-1579.

437 32. Zhang, T.; Wang, T.; Mejia-Tickner, B.; Kissel, J.; Xie, X.; Huang, C.-H. Environmental Science

438 \& Technology 2020, 54, (15), 9652-9661.

439 33. Huang, X.; Chen, X.; Chen, Q.; Yu, Q.; Sun, D.; Liu, J. Acta biomaterialia 2016, 30, 397-407. materials 2011, 192, (2), 786-793.

444 36. Liu, H.; Ni, X.-Y.; Huo, Z.-Y.; Peng, L.; Li, G.-Q.; Wang, C.; Wu, Y.-H.; Hu, H.-Y. Environmental 


\section{Supplementary Files}

This is a list of supplementary files associated with this preprint. Click to download.

- VideoS1.mp4

- VideoS2.mp4

- VideoS3.mp4

- VideoS4.mp4

- SI.pdf 